\title{
Orgasmic and ejaculatory problems in clinical practice
}

\author{
Stacy Elliott MD
}

$S$ Elliott. Orgasmic and ejaculatory problems in clinical practice. J Sex Reprod Med 2002;2(2):67-73.

Ejaculatory and orgasmic disorders are prevalent and distressing for many men with sexual concerns. They are best categorized into functional, neurological and anatomical problems, with the emphasis on whether the problem is presented as primarily a sexual or a medical concern for the patient. Ejaculatory and orgasmic concerns can present with, or be independent of, erection dysfunction. After physiology is explained, taking a sexual history and relevant physical examination issues are reviewed. An algorithm is described for distinguishing the disorders. Rapid or fast ejaculation can be managed by behavioural, pharmaceutical or medical methods. Delayed or inhibited ejaculation is more difficult to manage. The primary role of the family physician is to understand the differential diagnosis of these disorders, to not miss a reversible cause of them, and to refer those disorders that are difficult to assess and manage.

Key Words: Ejaculation; Orgasm; Sperm retrieval

\section{Troubles de l'éjaculation et de l'orgasme en pratique clinique}

Les troubles de l'éjaculation et de l'orgasme sont fréquents et s'avèrent source de stress pour la plupart des hommes qui éprouvent des difficultés sexuelles. On les classe en troubles fonctionnels, neurologiques ou anatomiques et il faut surtout voir si le problème est d'ordre sexuel ou d'ordre médical pour le patient. Les troubles de l'éjaculation et de l'orgasme peuvent être associés ou non au dysfonctionnement érectile. Une fois la physiologie expliquée, nous nous intéressons à la question des antécédents sexuels et de l'examen physique approprié. Suit un algorithme permettant de distinguer les différents troubles. L'éjaculation rapide ou prématurée peut se traiter par la thérapie comportementale, médicamenteuse ou médicale. Par contre, l'éjaculation retardée ou inhibée est plus difficile à traiter. Le rôle de l'omnipraticien consiste principalement à comprendre le diagnostic différentiel de ces troubles afin de ne pas laisser échapper une cause réversible et de confier à des spécialistes les cas difficiles à évaluer et à traiter.
W hile erectile dysfunction (ED) is the sexual disorder that most often brings men into the physician's office (1), ejaculatory and orgasmic concerns are an overlooked and under-reported area of male sexual functioning. Of reported sexual problems, the prevalence of ejaculatory and/or orgasmic disorders approaches $40 \%(2,3)$, with premature ejaculation being the most common disorder. In busy clinical practice, the 'sexual concerns' of the male patient are often presumed to be related to erection difficulties and intercourse mechanics, when, in fact, a complete, brief sexual history may uncover another etiology, such as ejaculation, sexual disinterest or pain. For example, premature ejaculation (and consequential detumescence) is often interpreted as ED by the patient, and can lead to a misdiagnosis and inappropriate treatment if a proper sexual history is not done.

Most men present to a primary care practice with sexual, versus more medical or organic, complaints related to their ejaculatory or orgasmic function. In general, younger men complain of fast or premature ejaculation, whereas older men present with delayed (or the inability to reach) ejaculation. In a study by Laumann et al (4), regardless of age, the prevalence of men who felt that they climaxed too early was upwards of $30 \%$, whereas the prevalence of those who were unable to achieve orgasm was less than $10 \%$. Other men present to the physician primarily with ejacula-

Vancouver Hospital and GF Strong Rehabilitation Center; Vancouver Sperm Retrieval Clinic; Division of Sexual Medicine,

Department of Psychiatry and Division of Urology, Department of Surgery, University of British Columbia; International Collaboration of

Repair Discoveries (ICORD), Vancouver, British Columbia

Correspondence: Dr Stacy Elliott, British Columbia Center for Sexual Medicine, Echelon - 5, 855 West 12th Avenue, Vancouver,

British Columbia V5Z 1M9. Telephone 604-875-8282, fax 604-875-8249, e-mail elliott@icord.org 
tory concerns of a medical nature, such as infertility, pain with ejaculation or hematospermia. It has been stated that only $2 \%$ of men who presented for treatment of subfertility were diagnosed with ejaculatory dysfunction as the cause of the subfertility (5).

When a patient has an orgasmic or ejaculatory concern, it is most helpful for the physician to place it into one of three categories that dictate different therapeutic streams:

- functional

- neurological

- anatomical

Functional problems are those that present as sexual dissatisfactions in men who are usually intact neurologically. They are often either issues with the timing of ejaculation (fast or premature ejaculation, or inhibited or delayed ejaculation) or concerns about the quality of the ejaculation or orgasmic experience (ie, decreased orgasmic sensation, poor ejaculate force or altered ejaculate volume).

Neurological disorders consist of either central or peripheral neurological interruptions in the two-phase ejaculatory process of seminal emission and propulsatile ejaculation. Most are caused by traumatic injury to the nerves (spinal cord injury or abdominal and/or pelvic surgery) or neurogenical disease processes (multiple sclerosis, diabetes mellitus and other peripheral neuropathies). Orgasm is often affected adversely.

Anatomical disorders may be congenital or acquired, usually interfere with ejaculation more than orgasm, per se, and present most often to the physician as infertility (ie, obstructive azoospermia) or ejaculatory pain.

Sexual neurophysiology dictates that erection is primarily a parasympathetic phenomenon, and ejaculation a sympathetic phenomenon, although neither process is that simple. Detumescence following ejaculation is due to the activation of the sympathetic nervous system. Most neurologically intact men undergo a sequential process of sexual arousal, penile tumescence and rigidity, and ejaculation accompanied by orgasm. Erection, ejaculation and orgasm, while related, are separate neurological events and can occur alone or out of sequence, especially in pathological conditions. For example, men with generalized ED secondary to surgery often discover that continued manipulation of a flaccid or semierect penis can lead to orgasm, with or without the expulsion of semen. It is, therefore, more useful to assess ejaculatory and orgasmic concerns as an entity that is separate from erection, while remaining cognizant of any changes to sexual interest or erection that may further clarify the etiology of the ejaculatory or orgasmic concern. Treatments of ejaculatory and orgasmic concerns are often coincidental with treatment of concomitant ED because the reduction of anxiety that occurs with reliable erectile ability allows for improved sexual satisfaction.

To better understand the category into which the clinician can place the patient, the physiology and definitions of sexual dysfunctions should be reviewed.

\section{ORGASM AND EJACULATION: WHAT IS THE DIFFERENCE?}

\section{Ejaculation}

Ejaculation is, basically, the complex process of sperm transport from the epididymis to the urethral meatus, resulting in the expulsion of semen. Ejaculation usually results in penile detumescence, after which a refractory period then ensues where arousal and/or orgasm is not possible again until a specific amount of time has passed. Both time for detumescence and the refractory period are age- and medicationdependent. Ejaculation may occur with or without an orgasm and/or erection. Both erection and ejaculation are spinal reflexes that are dependent on the removal of supraspinal (cerebral) inhibition for their triggering (6).

Ejaculation occurs in two phases: seminal emission and propulsatile ejaculation. Seminal emission is the process of sperm transport from the epididymis to the prostatic urethra, followed by closure of the bladder neck, and is under sympathetic control. Promoted by high sexual arousal, this phase has an element of voluntary control by the man. The engorgement of the prostate from vasocongestion of arousal emits pleasant afferent signals leading to preorgasmic sensations. The seminal vesicles and the prostate contribute the bulk of the seminal fluid, and the seminal bolus is delivered to the ejaculatory ducts. With concomitant and intermittent closure of the external sphincter and entrapment of the seminal bolus in the prostatic urethra, increased intraprostatic pressure results. Men recognize this is as a feeling of 'no return', or ejaculatory inevitability. This point, assumed previously to be the result of distention of the posterior urethra by the seminal bolus, must have additional afferent triggers, as radical prostatectomy patients can attest. In any case, the second phase of ejaculation, propulsatile ejaculation, is triggered. Propulsatile ejaculation consists of seminal vesicle, prostatic, urethral and pelvic floor muscle contractions that propel the seminal bolus out the end of the urethral meatus with considerable force. This is mediated by both parasympathetic and somatic input, and there is virtually no voluntary control of this phase. Disease, trauma or medications that interfere with the specific components of the autonomic or somatic nervous system have predictable results on the respective phases of ejaculation.

\section{Orgasm}

Orgasm is not as well defined neurologically as is ejaculation. It has been variously interpreted as a central interpretation of the genital events of ejaculation as well as a learned, local reflex with afferent (peripheral and cerebral) and efferent (sacral) limbs. Orgasm does not require intact genitalia (as seen with prostate removal and transsexual penectomy), and may be triggered by cerebral afferents alone (as in sleep and tantric sex experiences). Orgasm likely involves an intricate coordination of several nervous systems with hormonal modulation (6-9). A limited amount of testosterone appears to be crucial for attainment of orgasmic release. It has been noted that with conscious sexual arousal, orgasm may occur with damage to the sympa- 
thetic ganglia, but does not usually occur when there has been damage to the pudendal nerve (10). Orgasm may vary in intensity and duration, depending on many biopsychosocial factors such as duration of arousal, muscular tone of the pelvic floor, time lapse from last orgasm, general health, drug or alcohol consumption, intensity of arousal, and emotional intimacy and/or comfort with the partner.

\section{CLINICAL DEFINITIONS OF EJACULATORY AND ORGASMIC CONCERNS}

\section{Sexual dysfunctions}

Timing concerns: Fast or premature ejaculation is defined as ejaculation before or very soon after intromission that causes personal distress: this disorder is often considered psychogenic in nature. Inhibited or delayed ejaculation is defined as difficulty reaching ejaculation or orgasm despite long and adequate stimulation (as seen with the side effects of some antidepressant medications, in hypogonadal states and with neurological problems).

Ejaculation without orgasm: Often referred to as anhedonic ejaculation, the ejaculatory process occurs with very little sensation of seminal fluid passage and/or weak or absent orgasmic sensation. This is usually associated with neurological disease (multiple sclerosis and peripheral neuropathy) or, occasionally, psychiatric disorders.

Orgasm without antegrade ejaculate: Orgasmic sensation that is not accompanied by antegrade ejaculation (aspermia) is due to three possibilities: failure of seminal emission, interruption of the pathway for seminal transport or retrograde ejaculation into the bladder. A loss of seminal emission can occur with interruption with the sympathetic outflow to the pelvic plexus (non-nerve sparing retroperitoneal lymph node dissection, or abdominal, colon or pelvic surgery). 'Dry orgasm' usually refers to a postprocedural result following the removal of semen-producing organs or the loss or blockage of seminal pathways (ie, surgery or radiation to the prostate). Retrograde ejaculation is seen most often in men with surgical damage to the bladder neck (postsphincterotomy) or neurogenically impaired closure of the bladder neck (ie, diabetic neuropathy) where the ejaculate is expelled backwards into the bladder. Cloudy or frothy urine after orgasm is the typical sign of retrograde ejaculation.

Altered orgasmic quality: The subjective experience of orgasm can be altered significantly by medical and surgical conditions. This can be very distressing to patients and, unfortunately, is very difficult to manage.

Sexual dissatisfactions: Sexual dissatisfactions typically include self or partner concerns about the volume of seminal or pre-ejaculate fluid, reduced ejaculatory force, etc. Very little can usually be done to alter the etiology of these dissatisfactions, so management is symptom focused.

\section{Medical or organic ejaculatory concerns}

Even though these may present as medical versus sexual problems, it is important to note that these concerns usually have sexual repercussions.
Inability to ejaculate: Men who are not able to undergo seminal emission or propulsatile ejaculation are considered anejaculatory. The etiology is almost invariably neurogenic, and men with spinal cord injuries are the best example. Sperm retrieval methods are required for fertility. Aspermia (when ejaculation takes place without an antegrade ejaculate being produced) and azoospermia (an ejaculate that is void of spermatozoa) are different clinical entities from anejaculation.

Low volume or discoloured ejaculate: A low volume ejaculate may alter fertility potential, and the cause needs to be investigated. Discolouration of the semen is usually due to prostatic blood: persistent hematospermia needs urological workup.

Dissociated orgasm and ejaculation: When orgasm is experienced and ejaculatory fluid is expelled at a distinctly later time (via gravity), a neurogenic cause can usually be identified.

Ejaculatory pain: This pain is most often the result of an inflamed pathway (acute prostatitis, for example). It may also be referred pain or part of a complex pelvic pain syndrome, which requires a specialist to assess and manage.

\section{ASSESSING COMMON EJACULATORY AND ORGASMIC PROBLEMS}

The role of the physician is twofold. The first is not to miss a reversible cause of ejaculation or orgasmic dysfunction, and the second is to provide a differential diagnosis that will dictate further investigations and/or referral. Because of the complexity of these issues, the primary care physician cannot be expected to be an expert in the treatment of these disorders. However, he or she can undertake a proper assessment, alter those factors that may be contributing to the dysfunction, and manage patients to their professional level of comfort and expertise.

In assessing ejaculatory and/or orgasmic concerns, the distinction between entities is made from looking at the individual central and peripheral components as well as the capacity for inhibitory release. This understanding is required to differentiate whether the concern is functional, neurological or anatomical, and to assist in the formulation of an investigative and treatment strategy. For example, addressing abnormalities of the inhibitory release mechanism is the key to treating the functional difficulties (although the physiological component of functional problems must also be addressed). Anatomical and neurological orgasmic and/or ejaculatory difficulties require adaptation to, or overcoming of, the additional organic pathology with various medical or operative options, as well as dealing with the consequential secondary sexual component.

A full sexual and medical history and physical examination are necessary in the assessment of these concerns.

\section{The sexual history}

The questions below should be directed toward eliciting the patient's chief complaint.

1. Is this a sexual complaint (ie, is it interfering with 
sexual satisfaction, relationships and/or quality of life)?

2. Is the ejaculatory concern a cause of, or a contributing factor to, infertility?

3. Are there pain, blood or other medical and/or organic concerns with the process of ejaculation?

Sexual complaints are the most common, although they may overlap with infertility or medical concerns. If the complaint is sexual, patients often have difficulty articulating the difference between ejaculation and orgasmic concerns, and may well have both.

The next task is to clarify and classify the patient's chief complaint. Figure 1 may be helpful in directing the remaining queries. Providing simple definitions of ejaculation and orgasm may be helpful (ie, "Do you reach a point where you are very highly aroused and fluid comes out the end of your penis?" "Do you experience a very pleasant feeling of sexual release at any time?"), especially if the patient uses lay words such as "when I come".

Once the problem has been identified, a more detailed description will help unveil the etiology.

- Duration: How long has the problem been present?

Did it start abruptly or has it been gradual?

- Situation or generalized: Is the difficulty always present, or only in certain situations (with a partner, alone with attempts at masturbation, during the day and/or at night)? Depending on the problem, physicians may ask whether the problem improves or worsens with particular sexual acts (hand, oral or intercourse stimulation).

- What is the rest of the man's sexual response (desire, erection, freedom from sexual pain)?: Is the ejaculation or orgasmic concern tied to another problem that maybe the primary sexual issue that was missed? For example, a man with delayed orgasm due to hypogonadism will also have low sexual desire, small ejaculate volumes and a loss of morning erections (he may also have ED). Not asking these questions may cause the physician to miss a reversible medical cause of the patient's orgasmic disorder.

- What is the effect of the problem on the sexual relationship (sexual frequency, overall affection)?: Are there any sexual concerns of the partner? How has each partner reacted (positive, negative, blaming, supportive) to the problem? Part of the assessment should include the current sexual context, because successful therapy will depend on whether the physician's therapeutic intervention may be incorporated realistically into the man's sexual life. Is this a true sexual dysfunction or is it a symptom of relationship discord? If it is the latter, the couple needs counselling before any therapeutic intervention can begin.

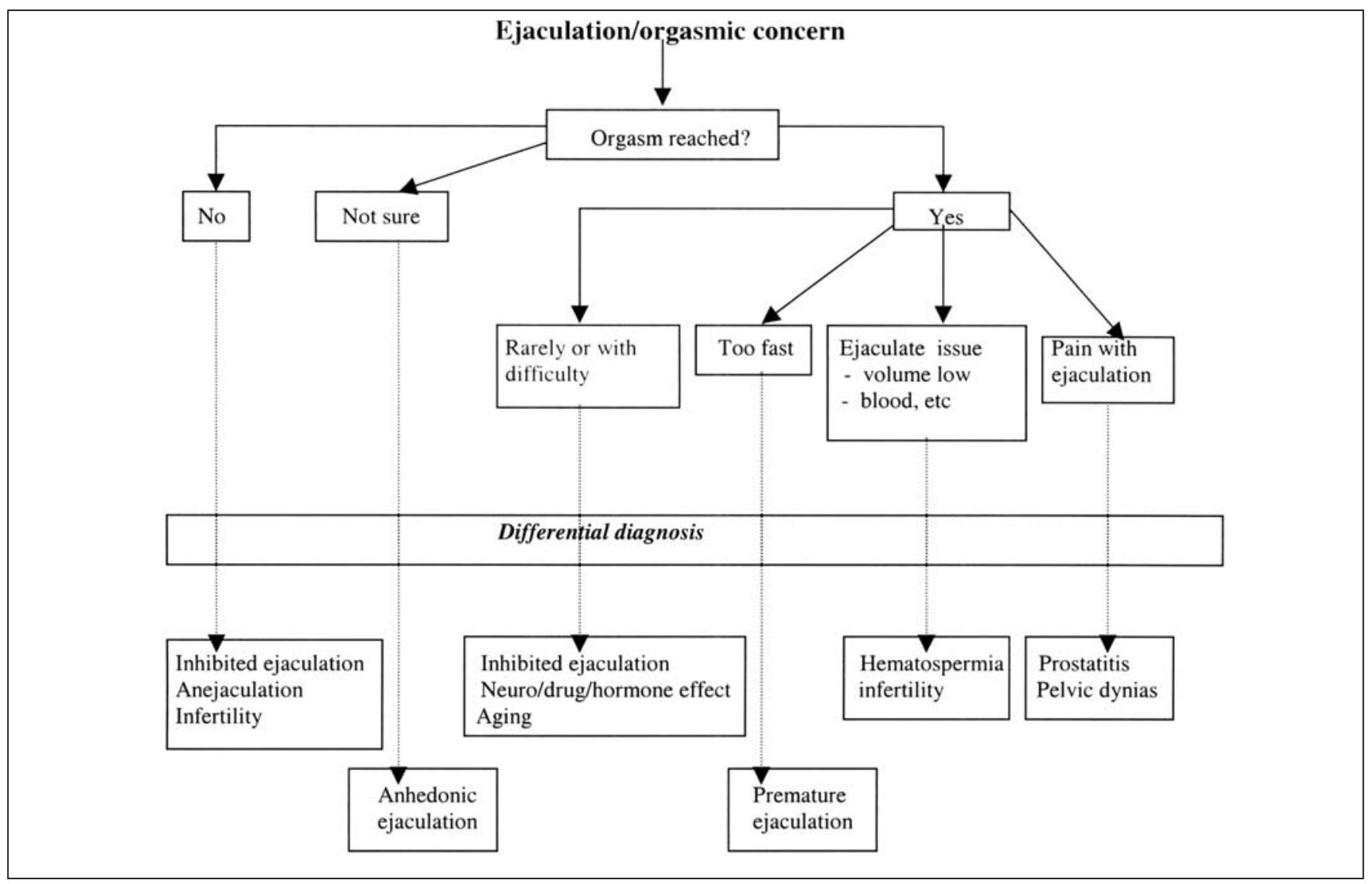

Figure 1) Differential diagnosis of ejaculatory and orgasmic concerns. Reproduced with permission from Elliott, S. Assessing orgasmic and ejaculatory problems in clinical practice. Medical Aspects of Human Sexuality, March 2001, page 27. Copyright 2001 Quadrant HealthCom Inc. All rights reserved 
- How motivated is the man (and his partner) to continue with investigations, therapy or referral?: Referrals to infertility specialists or other medical specialists may be necessary. If this is a functional issue that the physician feels is beyond his or her expertise, will the patient be open to seeing a qualified sex therapist?

\section{The medical history}

Medical conditions and drugs may interfere with ejaculation and orgasm. These influences, in turn, may interfere with sexual drive and sexual arousal, further compounding the problem. Both central and peripheral system disorders are well known to influence both orgasm and ejaculation. Complex neurotransmitters influence ejaculation; the neurotransmitter serotonin plays a central role. The serotoninselective reuptake inhibitor (SSRI) antidepressants are known for their ejaculatory-delaying side effect, as are other antihypertensive and antipsychotic medications (10). Dopamine is centrally prosexual, but there have been no studies to prove that dopaminergic drugs improve ejaculatory latency times, per se, in men. Because seminal emission is a sympathetic nervous system event, sympathomimetics with ephedrine-like action (eg, pseudoephedrine) are known to enhance the ejaculatory reflex.

\section{Physical examination and investigations}

The physical examination is necessary to rule out obvious anatomical and organic contributions. The physical examination is therapeutically beneficial, legitimizing the ejaculatory or orgasmic concern as a medical problem, and addresses concerns about sexually transmitted diseases and genital appearance.

A careful examination of the abdomen and genitalia needs to be done. Patency of the urethral meatus, size and firmness of the testicles, the presence or absence of spermatic cord structures, and foreskin retractability should be observed during the general genital overview. A rectal examination must be done to rule out prostatitis and other pathological conditions. Aside from testicular changes, additional hypogonadal body signs include the loss of body hair and muscle bulk, softening of the skin and gynecomastia. If a neurological cause is suspected, a neurological genital assessment is necessary. Sensory evaluation of the genital dermatomes, pinprick sensitivity of the glans, and rectal determination of anal tone and voluntary anal contraction are necessary. Intact pinprick sensation to the glans penis (signifying intact, upgoing, lateral spinothalamic spinal cord pathways) and voluntary anal contraction (demonstrating downgoing corticospinal pathways) are prognostic for the potential to experience genital orgasm (but do not rule out the potential for cerebrally generated orgasm). A positive bulbocavernosus reflex signifies an intact sacral reflex, which bodes well for ejaculation (and reflex erection) potential. Other signs of peripheral neuropathy or general cardiovascular compromise should be looked for.

In the field of ejaculatory disorders, investigations are gen- erally limited except for those involving ejaculatory pain and infertility. Ejaculatory pain must be investigated because there is usually an infectious (ie, prostatitis) or obstructive (stricture, stones, etc) cause. Infertility secondary to ejaculatory difficulties must be addressed in three stages: sexual ejaculatory capacity, volume of ejaculate, and semen quality of antegrade or retrograde specimens. Retrograde ejaculation can be assessed by looking for the presence of spermatozoa in the urine obtained after masturbation to orgasm. Serum hormone and gonadotropin levels may be assessed with the clinical suspicion of hypogonadism, hyperprolactinemia or fertility issues. Fertility issues need to be assessed by a urologist.

\section{MANAGING EJACULATORY AND ORGASMIC DISORDERS}

\section{Functional difficulties}

Functional difficulties stem from a lack of balance between the central brain control and the spinal cord reflexes. Orgasmic threshold is the critical level of input that allows ejaculation and orgasm to happen, and is constitutionally, but also situationally, determined. Poor control or oversuppression of the cerebral inhibition is the basis for most functional problems of premature ejaculation and delayed ejaculation, respectively. However, because ejaculation is primarily a sympathetic event, medications that are adrenergic or anticholinergic may enhance ejaculation by sensitizing the nerve endings involved in seminal emission, including closure of the bladder neck (11). The adrenergic tone that is generated by performance anxiety acts in the same way.

Fast or premature ejaculation: Many etiologies for fast or premature ejaculation have been suggested in the literature, including varied (and often weakly correlated) psychological causes. There is now a growing body of evidence that suggests organic factors may play a larger role. The latter include ejaculation reflex hyperexcitability due to impaired central inhibitory mechanisms, higher cortical representation of genital sensory stimuli and decreased penile vibratory thresholds (12). It seems reasonable to deduce that these factors are highly individualized, and account for the differences noted between lifelong and acquired, and primary and secondary fast ejaculators. In general, those men with lifelong, primary disorders are the most difficult to treat. Men with fast ejaculation are a heterogeneous population and must be assessed with a biopsychosocial model in mind.

The primary care physician can manage fast ejaculation through education (the ejaculatory process is biologically programmed and not abnormal, so it is a decision or wish to slow it down) and therapeutic intervention. The psychological intervention for fast ejaculation includes dealing with personal (guilt, fear and performance anxiety) and domestic (including partner communication) problems that can aggravate the heightened ejaculation reflex. Difficult problems need to be referred to a sex or marital therapist.

The medical management of fast ejaculation consists of a three-stream approach that may be used alone or in combination.

1. Reduce the reflex hyperactivity. 
2. Raise the orgasmic threshold pharmacologically (reversible).

3. Dampen the genital afferent signals of the ejaculatory reflex.

The basis of the behavioural approach, which was popularized in the 1950s, is 'retraining' the hyperactive reflex through physiotherapy-like exercises. Men with fast ejaculation do not appear to recognize the premonitory signs of impending ejaculation, and these exercises focus on this recognition, which ultimately improves physiological control. Masking these signs with products that dampen the afferent signals to the ejaculation reflex would be counterproductive. These techniques include self- or partnerstimulation (via gradated higher sexual stimuli) to the point of inevitability, at which point the ejaculatory urge is suppressed by either stopping the stimulus (stop-start technique) or by squeezing the penis directly beneath the glans (squeeze technique). Vaginal intromission and, finally, thrusting is allowed only when the ejaculatory control is improved. Continual and consistent exercises (minimum three times/week) in those who are well motivated usually results in increased ejaculatory latency, and the patient experiences improved self-esteem and continued motivation. Although follow-up of the original studies that purported success with these methods showed a decline in gain over time in the majority of cases, this has not been the universal clinical experience. Behavioural techniques aimed at altering the reflex itself, even in conjunction with pharmaceutical methods, are necessary for long term improvement, and may save the patient from pharmacological dependence in the long run. A helpful patient-directed book by Helen Singer-Kaplan, "PE: How to Overcome Premature Ejaculation" (13) is very useful when combined with weekly office visits to keep the patient encouraged.

Pharmacological methods, while successful in prolonging ejaculatory latencies (probably by increasing the orgasmic threshold), are dose-related and reversible when the drug is discontinued. The tricyclical antidepressant clomipramine hydrochloride (Anafranil, Novartis Phar-maceuticals, Canada) centrally inhibits 5-hydroxytryptamine (5-HT) reuptake, and the SSRIs effectively treat fast ejaculation. Either daily use or on demand use ( 2 to $6 \mathrm{~h}$ before intercourse) may be effective, but experimentation and individualization of dosing and timing are required; therefore, follow-up in the office is important. Clom-ipramine hydrochloride $25 \mathrm{mg}$ to $50 \mathrm{mg}$ is very effective, but has higher side effects than the SSRIs, so is best taken on an as needed basis. The SSRI paroxetine hydrochloride (Paxil, GlaxoSmithKline Inc, Canada) $20 \mathrm{mg}$ to $40 \mathrm{mg}$ once daily, sertraline hydrochloride (Zoloft, Pfizer Inc, Canada) $50 \mathrm{mg}$ to $200 \mathrm{mg}$ once daily, and fluoxetine hydrochloride (Prozac, Eli Lilly, Canada) $20 \mathrm{mg}$ to $40 \mathrm{mg}$ once daily, have also been tried on an as needed basis, but seem to be less successful when administered that way. It appears that paroxetine hydrochloride may be the most effective (12). The newer SSRIs have not been specifically tested. Erection enhancement methods (oral phosophodiesterase inhibitors or intracavernosal injections) have been used to treat fast ejaculation through the indirect secondary benefit of anxiety reduction when the erection concerns are managed. Evidence of a direct latency-producing effect by phosphodiesterase inhibitors used to treat ED is weak and has been found only in vitro.

The last method of managing fast or premature ejaculation, dampening the afferent signals, has been the method of desperation for many young men who seek to avoid recognizing the premonitory signals of ejaculation. The use of topical anesthetic creams and sprays or multiple condom barriers to decrease penile sensation, as well as purposeful avoidance of sexually arousing thoughts, serves to decrease the afferent arc of the reflex. While successful in the short term and for demanding individual situations, the conscious physical and mental distraction from physical and emotional pleasure is unsatisfying in the long term for both the man and his partner. Because the theory behind this method is the opposite of the behavioural method, in the end, it will serve only to reinforce the hypersensitivity of the reflex. It is the least effective method of long term management, but may be the only alternative if a short term solution is urgently required and the other alternatives are not acceptable.

Delayed ejaculation: Eight per cent of the men in the Laumann study (4) reported difficulty reaching orgasm, with men aged 50 to 53 years (14\%) showing the most difficulty (4).

Delayed ejaculation is difficult to manage. Reversible causes such as low testosterone, the use of medications that delay orgasm (especially the SSRIs) and other hormonal problems that depress sexual function (depression, hypothyroidism, hyperprolactinemia) should be ruled out. In younger patients, over-controlling the inhibitory mechanism is usually the problem. Neurology is usually intact, as evidenced by nocturnal emissions. The patient tries to compensate for this by overstimulating the genitalia (usually the erection is quite sustainable) while not being sexually aroused enough emotionally, which reinforces the original problem. Focusing on high mental arousal before adding genital stimulation, and learning to ejaculate to lighter genital stimulus (through self-stimulation exercises) is required. Often, referral to a sexual medicine expert or sex therapist is necessary, especially with lifelong primary inhibited ejaculation. In men with fertility agendas, referral to a sexual medicine physician or urologist specializing in sperm retrieval may be necessary (14). Older men who are not hypogonadal and who have acquired delayed ejaculation or are unable to ejaculate often report loss of penile sensitivity, which is an aging phenomenon. Usually, the pattern of sexual stimulation that has worked for many years is no longer adequate, and more genital stimulation is required. The physician can be instrumental in 'giving permission' to increase the stimulation within the respectful context of the established sexual relationahiop. Kegel exercises for men may improve the tone of the pelvic floor and assist with ejaculation. Pharmacological treatment of 
delayed ejaculation or orgasm secondary to antidepressant use includes the use of medications before intercourse that seek to counteract the prevalence of serotoninergic blockade, ie, bethanechol chloride ( $10 \mathrm{mg}$ to $20 \mathrm{mg}, 1$ to $2 \mathrm{~h}$ before intercourse) and cyproheptadine hydrochloride ( $4 \mathrm{mg}$ to $8 \mathrm{mg}, 1$ to $2 \mathrm{~h}$ before intercourse) (15). There is some anecdotal evidence that the use of 'sex friendly' antidepressants (bupropion hydrochloride $75 \mathrm{mg}$ once daily, nefazodone hydrochloride $150 \mathrm{mg} 1 \mathrm{~h}$ before intercourse), sympathomimetics such as pseudoephedrine $(30 \mathrm{mg}$ to $60 \mathrm{mg}$ or $120 \mathrm{mg}$ slow release) and even yohimbine hydrochloride $6 \mathrm{mg}$ to $18 \mathrm{mg}$ as needed, may be helpful in some cases of delayed ejaculation, but studies are not yet available. It is important to note that sympathomimetics cannot be used in hypertensive patients, and that yohimbine may cause hypotension.

\section{Neurological etiologies of ejaculatory and orgasmic disorders}

Neurological problems usually cause difficulties with activating the spinal reflex of ejaculation and may blunt or significantly alter the subjective response of orgasm. In rare cases, they can lower the orgasmic threshold, as seen with injuries to the lower thoracic, lumbar or sacral cord, which may cause spontaneous ejaculation or orgasm (16). A practical review of the sexual consequences of neurological conditions can be found in the article by Elliott (14). The management of neurological ejaculatory and orgasmic problems is difficult and should be referred to sexual rehabilitative medicine specialists. Additional information on the sexual consequences of neurological illnesses can be found in articles by Waldman and Elias (17) and Smeltzer and Kelly (18). Ejaculatory disorders that require sperm retrieval, such as vibrostimulation and electroejaculation, are elucidated well in the papers by Elliott and Fluker (19), Oates and Kasabian (20) and Ohl (21).

\section{REFERENCES}

1. Krane RJ, Goldstein I, De Tejada IS. Impotence. N Engl J Med 1989;321:1648-59.

2. Levine SB. Sexual Life: A Clinician's Guide: Critical Issues in Psychiatry. New York: Plenum Press, 1992.

3. Laumann EO, Paik A, Rosen RC. The epidemiology of erectile dysfunction results from the National Health and Social Life Survey. Int J Impot Res 1999;11(Suppl 1):S60-4.

4. Laumann EO, Paik A, Rosen RC. Sexual dysfunction in the United States: Prevalence and predictors. JAMA 1999;128:537-44.

5. Dubin L, Amelar RD. Etiological factors in 1294 consecutive cases of male infertility. Fert Steril 1971;22:469-74.

6. Marson L. Central nervous system control. In: Carson C, Kirby R, Goldstein I, eds. Textbook of Erectile Dysfunction. Oxford: ISIS Medical Media, 1999:73-88.

7. Alexander CJ, Sipski ML, Findley TW. Sexual activities, desire, and satisfaction in males pre- and post-spinal cord injury. Arch Sex Behav 1993;22:217-28.

8. Sipski ML, Alexander CJ, Rosen R. Sexual arousal and orgasm in women: Effects of spinal cord injury. Ann Neurol 2001;49:35-44.

9. Whipple B, Gerdes CA, Komisaruk BR. Sexual response to self-stimulation in women with complete spinal cord injury. J Sex Res 1996;33:231-40.

10. Rehman J, Melman A. Normal anatomy and physiology. In: Mulcahy JJ, ed. Male Sexual Function. Totowa: Humana Press, 2001:1-46.

11. Hendry WF. Causes and treatment of ejaculatory disorders. In: Carson C, Kirby R, Goldstein I, eds. Textbook of Erectile Dysfunction. Oxford: ISIS Medical Media, 1999:569-82.
Anatomical causes of ejaculatory and orgasmic disorders (11)

Abnormalities of ejaculatory function usually present to the physician as infertility. However, ejaculatory symptoms such as pain or hematospermia should always be investigated. Congenital causes of ejaculatory dysfunction include incomplete or altered embryological development of the epididymis, vas deferens, prostate, ejaculatory ducts or penis and urethra (hypospadias, epispadias and bladder extrophy). Acquired causes include those that are secondary to surgery or radiation that interfere with the anatomy or the nervous control of the ejaculatory system, and those that are secondary to infective processes causing pain or obstruction. Preliminary investigations (cultures, hormone evaluation and, if appropriate, semen analysis) and basic trials of appropriate therapies that the primary care physician feels comfortable with should be done before any referral.

\section{CONCLUSIONS}

Ejaculatory and orgasmic disorders are less prevalent than erection disorders in clinical practice, but may be just as distressing for the patient. They are most easily classified according to their functional, neurological or anatomical categories. Whether the concern is primarily a sexual, or a medical/organic issue, is delineated by the patient himself. The most common ejaculatory disorders seen in primary care are those of sexual or functional difficulties. The most prevalent one, premature ejaculation, is best assessed by using the biopsychosocial model. Inhibited ejaculation is less common than premature ejaculation. Neurological alterations resulting from various disruptions of the ejaculatory pathways result in orgasmic and ejaculatory reflex abnormalities. Anatomical ejaculatory problems usually present as infertility or ejaculatory pain. A full sexual history, medical history and physical examination are essential to not missing reversible and other etiological factors that will dictate further investigations and therapy.

12. McCullough AR. Ejaculatory disorders. In: Mulcahy JJ. Current Clinical Urology: Male Sexual Function: A Guide to Management. Totowa: Humana Press, 2001:351-70.

13. Kaplan HS. PE: How to Overcome Premature Ejaculation. New York: Brunner/Mazel, 1989.

14. Elliott S. Assessing orgasmic and ejaculatory problems in clinical practice. Med Aspect Hum Sex 2001;3:26-30.

15. Maurice W. Sexual Medicine in Primary Care. St Louis: Mosby Inc, 1999.

16. Kuhr CS, Heiman J, Cardenas D, Bradley W, Berger RE. Premature emission after spinal cord injury. J Urol 1995;153:429-31.

17. Waldman TL, Elias B. Cancer. In: Sipski M, Alexander C, eds. Sexual Function in People with Disability and Chronic Illness. Gaithersburg: Aspen Publishers, 1997:337-54.

18. Smeltzer SC, Kelly CL. Multiple sclerosis. In: Sipski M, Alexander C, eds. Sexual Function in People with Disability and Chronic Illness. Gaithersburg: Aspen Publishers, 1997:177-88.

19. Elliott SL, Fluker MR. Fertility options for men with ejaculatory disorders. J SOGC 2000;1:26-32.

20. Oates RD, Kasabian NG. The use of vibratory stimulation for ejaculation abnormalities. In: Whitehead D, Nagler H, eds. Management of Impotence and Infertility. Philadelphia: JB Lippincott, 1994:314-20.

21. Ohl DA. The use of electroejaculation for ejaculation abnormalities. In: Whitehead D, Nagler H, eds. Management of Impotence and Infertility. Philadelphia: JB Lippincott, 1994:294-313. 